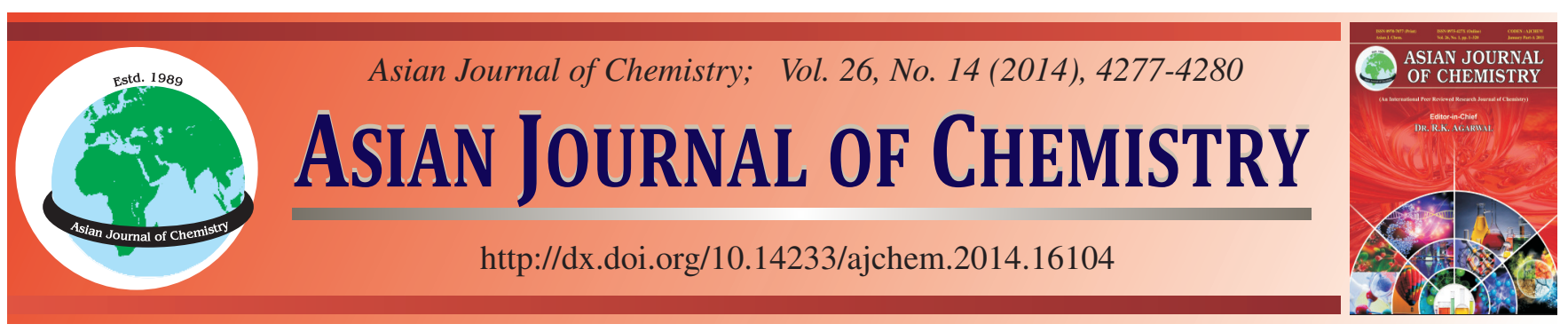

\title{
Analysis of Aconitine Metabolites in Ten-Year Aconitine Poisoning Human Liver Sample Using High Performance Liquid Chromatography-Tandem Mass Spectrometry
}

\author{
Yong-Gang Liu ${ }^{1}$, Qun-Shu Zhang ${ }^{2}$, Ying-Qiang Lu², Min Zuo ${ }^{4}$, Guo-Zhong Zhang ${ }^{4}$ and Hong-Gui Zhang ${ }^{1, *}$
}

\author{
${ }^{1}$ School of Chinese Materia Medica, Beijing University of Traditional Chinese Medicine, Beijing 100102, P.R. China \\ ${ }^{2}$ School of Pharmacy of Shenyang Pharmaceutical University, Shenyang 110016, P.R. China \\ ${ }^{3}$ Laboratory of forensic Medicine of Jilin University, Changchun 130021, P.R. China \\ ${ }^{4}$ Hebei Medical University, Shijiazhuang 050017, Hebei Province, P.R. China
}

*Corresponding author: E-mail: zhanghg4@163.com

\begin{abstract}
Described here is a fatal case of accidental aconitine poisoning in a patient who took traditional Chinese medicine (TCM) formula containing radix aconite, radix aconite laterals preparation and radix aconite kusnezoffii ten years ago. The patient fell into a coma and died of brain edema $5 \mathrm{~h}$ after diagnosis. Consent for autopsy was confirmed by the family, the toxicity of aconitine alkaloids in the liver was analyzed by LC-MS. According to MS/MS and MS3 spectra data, three metabolites were identified as aconitine (A), benzoylaconine (B) and 16-O-demethylaconitine (C), respectively through comparison with authentic substances and among them, 16-O-demethylaconitine was reported for the first time. Metabolic pathway of aconitine in human liver was proposed. In this paper, the test sample is the longest storage time of all reported samples, so the result is useful to solve many long unsettled law cases of accidental or intentional aconitine poisoning which were caused for obsolete method.
\end{abstract}

Keywords: Liquid chromatography-mass spectrometry, Aconitine, Metabolite, 16-O-demethylaconitine, Aconitine poisoning.

ᄂ _ - _ - - - - - - - - - - - - - - - - - - - - - - - - - -

\section{INTRODUCTION}

Ten years ago, a 34-year-old female took a traditional Chinese medicine formula containing aconite. Soon, she developed nausea, diarrhea, numbness of the upper and lower extremities and discomfort of the entire body. Her consciousness and response were relatively clear and normal on being sent to hospital. In accordance with her complaint and explanation, aconitine poisoning was strongly suspected and the gastric lavage with activated charcoal was performed immediately. However, she was still dead after poisoning. Due to apparatus and method limitation and aconitum alkaloids are very unstable and decomposed fast in human body ${ }^{1-3}$, aconitine poisoning was confirmed according to the patient's symptoms ten years ago. Based on a lot of preliminary work about aconitine in our laboratory ${ }^{4-7}$, a new method was established in our lab. Therefore, a request for an autopsy was consent and her liver was obtained for aconitine alkaloid analysis by LC-MS ${ }^{\mathrm{n}}$. In this paper, three compounds were identified and among them, 16O-demethylaconitine was reported for the first time.

\section{EXPERIMENTAL}

Formic acid and acetonitrile were of HPLC grade (Yuwang Co., Shandong, China). All other chemicals used were of analytical reagent grade and charged from Beihua Fine Chemicals
Co., Ltd. (Beijing, China), the water used was distilled water. Solid phase extraction (SPE) C18 Cartridges were obtained from Waters (Milford, MA, USA). The liver sample from a woman died of aconite intoxication was obtained from Department of Forensic Medicine of Hebei Medicine University (China) and stored in formalin until required for analysis.

The sample was analyzed by LC-ESI-MS ${ }^{\mathrm{n}}$ using a Thermo Finnigan LCQ ion trap liquid chromatography-mass spectrometers (San Jose, USA) equipped with an electrospray ion source, an autosampler and an LC-10AD pump (Shimadzu, Kyoto, Japan).

Methods: The HPLC instrument was (DAD), an autosampler and a column compartment. Samples were separated on an Agilent XDB C18 column $(150 \times 4.6 \mathrm{~mm}$ I.D., $5 \mu \mathrm{m})$. The mobile phase consisted of acetonitrile (A) and water containing $0.1 \%(\mathrm{v} / \mathrm{v})$ ammonia water (B). A gradient program was used as follows: $0 \mathrm{~min}, 2: 98(\mathrm{~A} / \mathrm{B}, \mathrm{v} / \mathrm{v}) ; 5 \mathrm{~min}, 5: 95 ; 10$ $\min , 25: 85 ; 30 \mathrm{~min}, 40: 60 ; 50 \mathrm{~min}, 80: 20$. A 15 min post-run time was set to fully equilibrate the column. The flow rate was $1 \mathrm{~mL} / \mathrm{min}$. The column temperature was $25^{\circ} \mathrm{C}$. The samples were detected at $240 \mathrm{~nm}$. The sample injection volume was $10 \mu \mathrm{L}$. The HPLC eluent was introduced into ESI source of mass spectrometer in a post column splitting ratio of $4: 1$. ESIMS was performed in the positive mode and the spray voltage 
was set at $4.50 \mathrm{kV}$. The capillary voltage was adjusted at 6.25 $\mathrm{V}$ and its temperature was maintained at $200{ }^{\circ} \mathrm{C}$. The sheath gas $\left(\mathrm{N}_{2}\right)$ flow-rate was set at 70 a.u. and the auxiliary gas flowrate was at 10 a.u. The alkaloids were detected in full-scan and selected reaction monitoring (SRM) modes according to the method reported previously ${ }^{7}$ with a small modification. $\mathrm{MS}^{2}$ and $\mathrm{MS}^{3}$ spectra were acquired to confirm the alkaloids' structures further.

Isolation of metabolites: The liver was smashed and then extracted with water. After being deproteinized, $1 \mathrm{~mL}$ of aliquot was filtered through cellulose membrane $(0.45 \mu \mathrm{m})$ and directly applied to SPE C18 cartridge pre-equilibrated with $3 \mathrm{~mL}$ of distilled water, $3 \mathrm{~mL}$ of methanol and $3 \mathrm{~mL}$ of distilled water, sequentially. The cartridge was washed with $2 \mathrm{~mL}$ of distilled water and eluted with $1 \mathrm{~mL}$ of methanol, respectively. The eluate was evaporated to dryness gently under a stream of nitrogen at $40{ }^{\circ} \mathrm{C}$, then the residue was dissolved in $100 \mu \mathrm{L}$ of the mobilephase and a $50 \mu \mathrm{L}$ aliquot was injected into the chromatographic system. Separation was performed with a BDS-C18 column (particle size $5 \mu \mathrm{m}, 150 \times 4.6 \mathrm{~mm}$ I.D, Dalian Elite Analytical Instrument Co. Ltd., Dalian, China) at room temperature, the mobile phase was acetonitrile-water-formic acid (40:60:0.5, v/v/v) and the flow-rate was $0.4 \mathrm{~mL} / \mathrm{min}$.

\section{Clinical findings}

Organ change analysis: Stomach: The colour of gastric inclusion was dust-colour, the stomachic mucosa showed focal petechial hemorrhage which distributed mainly gastric pylorus, bottom and body of stomach. Brain: pial arteriole in brain expanded slightly. The others were normal.

Tissue change analysis: Brain: Molecular layer of cerebral cortex loose; edema was seen around the collateral blood capillaries, neural cell and venule, change in cell number followed an autolysis. Leukoaraiosis in brainstem, neurons of the nucleus of brain showed autolysis change. Heart: neural cell swelled, nissel bodies appeared; purkinje cells lysis, small vessels of the substantia alba were dilated, congestive and had a evident rarefaction and hydroncus. Lung: muscle fibers fractured with irregular edge. Hypertrophy and atrophy are all coexistence in fiber cell. Evident thickening of the alveoli of lung wall, Micrangium were dilated, congestive and hydroncus. Liver: veinlet and antrum of liver were expanded, gored.

\section{RESULTS AND DISCUSSION}

Three compounds were found in the liver sample, including aconitine and its two metabolites, bezoylaconine (A) and 16-O-demethylaconitine $(\mathrm{C})$. The structures of the compounds are shown in Fig. 1, which were identified by comparison of LC retention time, MS/MS and $\mathrm{MS}^{3}$ spectra with that of authentic sample in rabbit urine reported previously.

Acetonitrile gave a LC retention time of $26.79 \mathrm{~min}$ and the pseudomolecular ion $[\mathrm{M}+\mathrm{H}]^{+}$was at $m / z, 646$ as shown in Fig. 2. SRM used precursor $\rightarrow$ product ion combination at $m / z$ $646 \rightarrow m / z$ 586. It also displayed $\mathrm{MS}^{2}$ spectrum of fragment

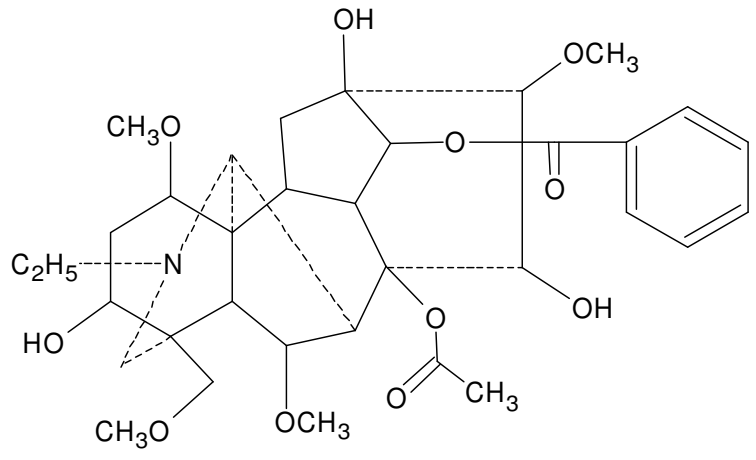

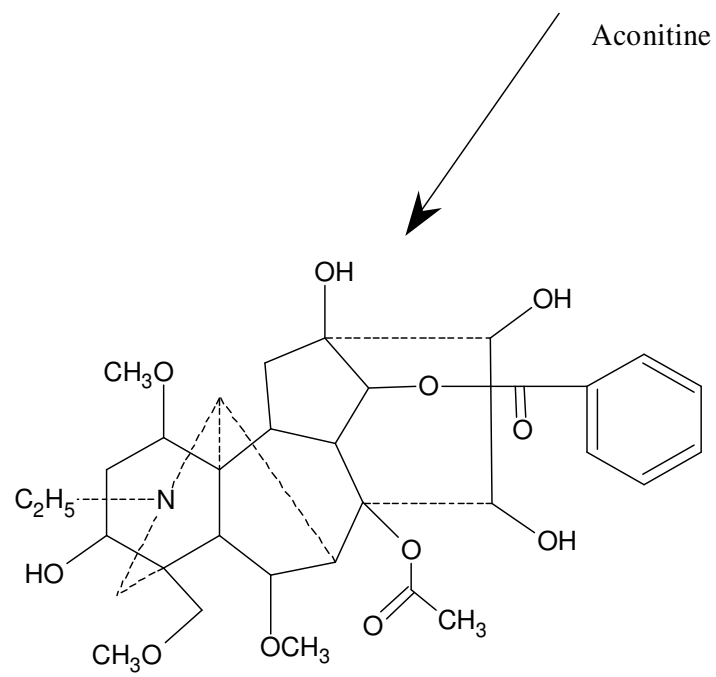

16-O-Demethylaconitine (C)

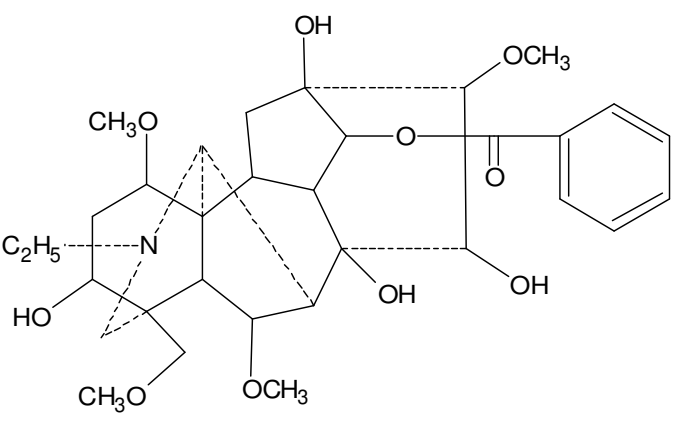

Benzoylaconine (B)

Fig. 1. Structures of aconitine, its metabolites and proposed metabolic Pathways in human body 

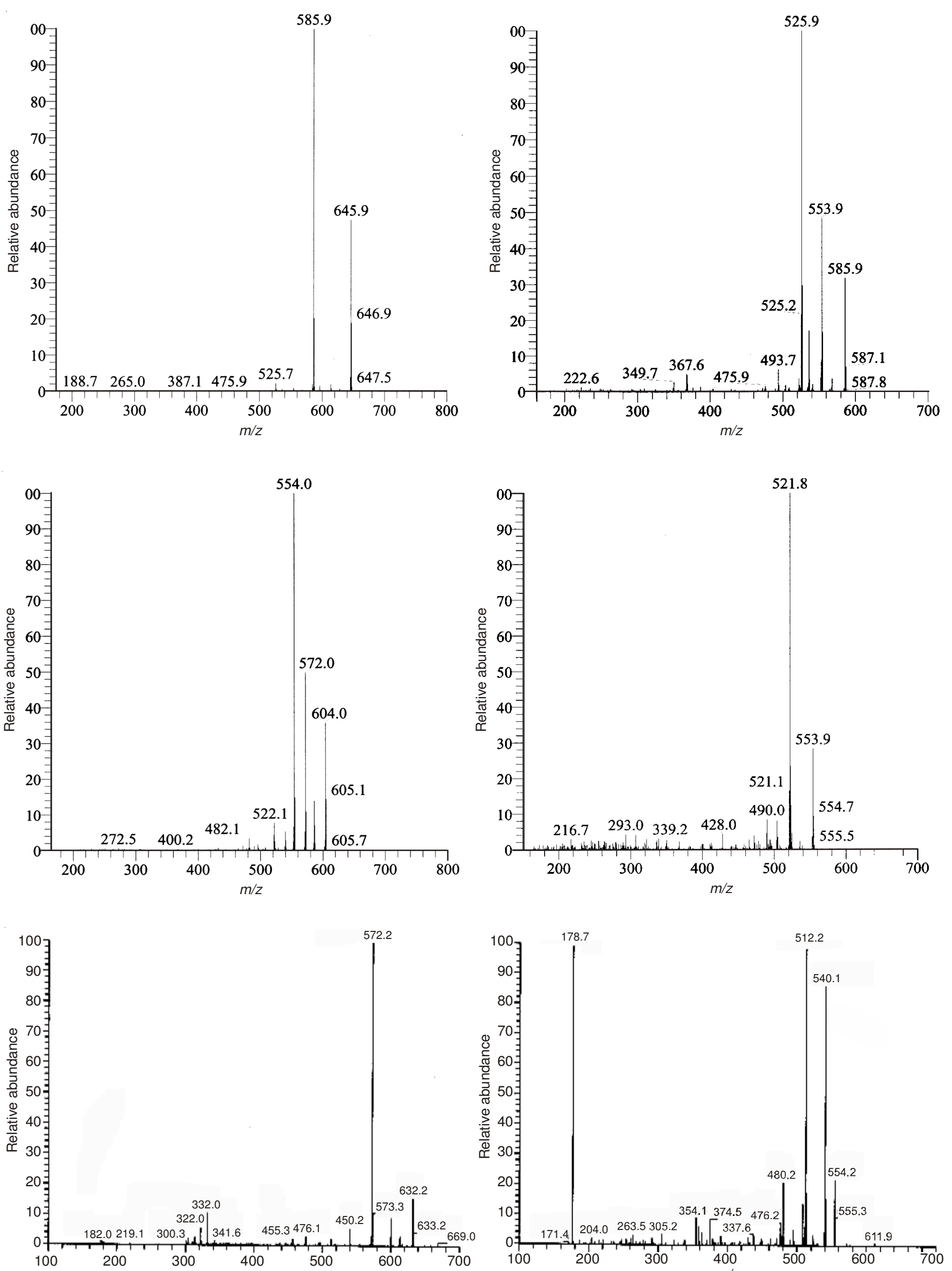

Fig. 2. MS/MS and $\mathrm{MS}^{3}$ spectra of the aconitine and its metabolites 
ions at $m / z, 586,554$ and 526, resulting from loss of $\mathrm{CH}_{3} \mathrm{COOH}$ $(60 \mathrm{u}), \mathrm{CH}_{3} \mathrm{OH}(32 \mathrm{u})$ and $\mathrm{CO}(28 \mathrm{u})$, respectively. Further detected by $\mathrm{MS}^{3}$ spectrum of fragment ions at $\mathrm{m} / \mathrm{z}, 554,526$ and 494. It suggested that $\mathrm{A}$ was aconitine, referring to the metabolites in rabbits urine . $^{8}$.

The $\mathrm{LC}$ retention time of $\mathrm{B}$ was $12.05 \mathrm{~min}$ and pseudomolecular ion $[\mathrm{M}+\mathrm{H}]^{+}$was at $m / z, 604$ as shown in Fig. 2. The $\mathrm{MS}^{2}$ spectrum of B showed a number of fragment ions at $\mathrm{m} / \mathrm{z}$ 586, 572 and 554 owing to loss of $\mathrm{H}_{2} \mathrm{O}(18 \mathrm{u}), \mathrm{CH}_{3} \mathrm{OH}(32 \mathrm{u})$ and both of them. The $\mathrm{MS}^{2}$ spectrum of $\mathrm{B}$ was similar to that of $\mathrm{A}$ and referring to the metabolites in rabbits' urine ${ }^{9}$, B was identified as benzoylaconine.

C showed a LC retention time of $19.25 \mathrm{~min}$ and pseudomolecular ion $[\mathrm{M}+\mathrm{H}]^{+}$at $m / z, 632$ as shown in Fig. 2. $\mathrm{MS}^{2}$ spectrum displayed the fragment ion at $\mathrm{m} / \mathrm{z}, 614,572,540$ and 512, resulting from loss of $\mathrm{H}_{2} \mathrm{O}(18), \mathrm{CH}_{3} \mathrm{COOH}(60 \mathrm{u})$, $\mathrm{CH}_{3} \mathrm{OH}(32 \mathrm{u})$ and $\mathrm{CO}(28 \mathrm{u})$, respectively. The $\mathrm{MS}^{2}$ spectrum of $\mathrm{C}$ was similar to that of $\mathrm{A}$, the same as 16-O-demethylaconitine. Combined the metabolic pathways of lappaconitine $^{10}, \mathrm{C}$ was identified as $16-\mathrm{O}$-demethylaconitine.

In this case, study was focused on compounds from traditional Chinese medicine (TCM) formula and their metabolites in human liver, though it was difficult to separate and identify these compounds in biological sample. As described in Experimental, the liver sample from a woman died of aconite intoxication was obtained from Department of Forensic Medicine of Hebei Medical University (China) in 2003 and stored in formalin until required for analysis. It was the first time that aconitine and its two metabolites were identified in such biological sample by LC-MS ${ }^{\mathrm{n}}$. It suggested that LC-MS was very sensitive in identification of trace compounds in complex biological sample. The detection limit of Aconitum alkaloids was $1 \mathrm{ng}$.

All of the three compounds reported in this paper are alkaloids which were identified by comparing retention time, pseuomolecular ion $[\mathrm{M}+\mathrm{H}]^{+}$and other ion signal peaks with those authentic samples. Aconitine was further identified by $\mathrm{MS}^{3}$ spectra, which could give more information of the structure. The retention time of all alkaloids are described above. With BDS $\mathrm{C}_{18}$ column, the retention time was longer if the polarity of mobile phase was lower.

Compared with parent Aconitum alkaloids, the metabolites only changed on side chains, suggesting that the toxicity was due to the ester of C- 8 group. The compounds by hydrolysis at the $\mathrm{C}-8$ acetyl ester $(\mathrm{B})$ is much less toxic than the parent $\operatorname{drug}^{9}$ (A). When the C-8 and C-14 groups were both hydrolyzed, the aconine was almost without atoxic ${ }^{9}$ and this paper provides information of their chemical structures. The result is useful for researches on Aconitum alkaloids poisoning and metabolic detoxification.

Aconitum alkaloids poisoning was usually confirmed by detecting parent Aconitum alkaloids in human body. But these alkaloids decomposed rapidly, it is difficult to detect them in human body. The metabolites found in human liver can testify the Aconitum alkaloid absorbed and provide chemical information for identification of Aconitum alkaloids poisoning. The metabolites of Aconitum alkaloids have been known, so to confirm Aconitum alkaloids poisoning in forensic medicine, determination of the parent Aconitum alkaloids can be replaced by their metabolites now. The structures of Aconitum alkaloids and their metabolites and the proposed metabolic pathways of Aconitum alkaloids in human are shown in Fig. 1. Three alkaloids were identified firstly in biological sample contained compounds from traditional Chinese medicine formula and $\mathrm{C}$ was identified for the first time.

This aconitine poisoning sample from a personal liver kept in formalin for four years was detected by this method, the result was credible and duplicate. This method was applied to detect a aconitine poisoning sample which was obtained several years ago. So, it can be used in insoluble case of accidental or intentional aconitine poisoning before. This new technique provide a new way to solve many unsettled law cases which were caused for obsolete method and detecting instrument.

\section{ACKNOWLEDGEMENTS}

We gratefully acknowledge financial support from the National Natural Science Foundation of China, No. 39870874; 31070328.

\section{REFERENCES}

1. Y.G. Wang, S.Q. Wang, Y.X. Liu, L.P. Yan, G.F. Dou and Y. Gao, J. Chromatogr. B, 844, 292 (2006).

2. G.G. Tan, Z.Y. Lou, J. Jing, W.H. Li, Z.Y. Zhu, L. Zhao, G.Q. Zhang and Y.F. Chai, Biomed. Chromatogr., 25, 1343 (2011).

3. H. Yue, Z.F. Pi, F.R. Song, Z.Q. Liu, Z.W. Cai and S.Y. Liu, Talanta, 77, 1800 (2009).

4. N. Yoshioka, K. Gonmori, A. Tagashira, O. Boonhooi, M. Hayashi, Y. Saito and M. Mizugaki, Forensic Sci. Int., 81, 117 (1996).

5. Y.G. Liu, P. Tan, F. Li and Y. Qiao, Drug Test. Anal., 5, 480 (2013).

6. P. Tan, Y.G. Liu, F. Li and Y.J. Qiao, Die Pharmazie, 67, 274 (2012).

7. H.G. Zhang, Y. Sun, M.Y. Duan, Y.J. Chen, D.F. Zhong and H.Q. Zhang, Toxicon, 46, 500 (2005).

8. H.G. Zhang, X.G. Shi, Y. Sun, M.Y. Duan and D.F. Zhong, Chin. Chem. Lett., 13, 758 (2002).

9. F.M. Xie, H.C. Wang, H.L. Shu, J.H. Li, J.R. Jiang, J.P. Chang and Y.Y. Hsieh, J. Chromatogr. B; Biomed. Sci. Appl., 526, 109 (1990).

10. H.X. Kuang, Traditional Chinese Medicine Chemistry, China Press of Traditional Chinese Medicine, Beijing, p. 361 (2003). 